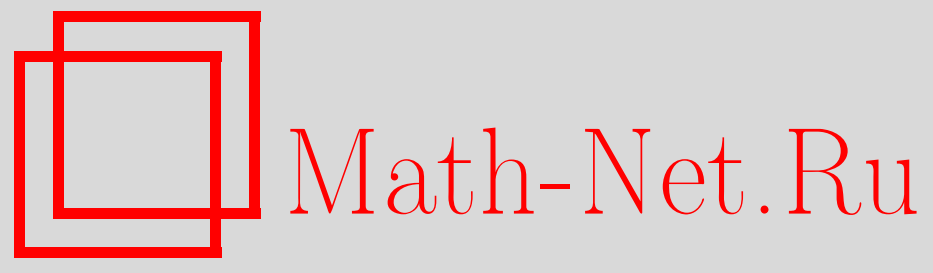

А. Н. Зарубин, Нелокальная краевая задача Трикоми для дифференциально-разностного уравнения смешанного типа, Вестн. Сам. гос. техн. унта. Сер. Физ.-мат. науки, 2021, номер 1, 35-50

DOI: https://doi.org/10.14498/vsgtu1835

Использование Общероссийского математического портала MathNet.Ru подразумевает, что вы прочитали и согласны с пользовательским соглашением

http://www . mathnet.ru/rus/agreement

Параметры загрузки:

IP: 34.227 .88 .159

26 апреля 2023 г., 18:12:55

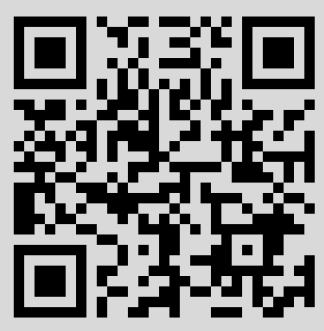


УДК 517.956.6

\title{
Нелокальная краевая задача Трикоми для дифференциально-разностного уравнения смешанного типа
}

\author{
(C) A. Н. Зарубин
}

Орловский государственный университет имени И. С. Тургенева, Россия, 302026, Орел, Улица Комсомольская, 95.

\section{Аннотация}

Исследуется краевая задача Трикоми для дифференциально-разностного опережающе-запаздывающего уравнения смешанного типа с некарлемановскими отклонениями по всем аргументам искомой функции. Применена редукция к уравнению смешанного типа без отклонений. Используются симметричные попарно коммутативные матрицы коэффициентов уравнения. Доказаны теоремы единственности и существования. Задача однозначно разрешима.

Ключевые слова: уравнение смешанного типа, дифференциально-разностное уравнение, интегральное уравнение, сингулярное интегральное уравнение, сосредоточенное запаздывание и опережение.

Получение: 5 ноября 2020 г. / Исправление: 13 февраля 2021 г. Принятие: 22 февраля 2021 г. / Публикация онлайн: 10 марта 2021 г.

Введение. Дифференциально-разностные уравнения (как обыкновенные, так и с частными производными, с сосредоточенным карлемановским или некарлемановским запаздыванием и опережением) служат математическими моделями для многих прикладных задач таких как, вихреобразование, перемежаемость, формирование сложных когерентных пятен [1]; многослойные оболочки и пластины [2]; плазма [3]; колебания кристаллической решетки [4]; проблема оптимизации лечения онкологических заболеваний [5].

Данная работа посвящена изучению краевой задачи Трикоми для нелокального уравнения смешанного типа Лаврентьева-Бицадзе с сосредоточенным запаздыванием и опережением по всем аргументам искомой функции вида

\section{Научная статья}

(2)(7) Контент публикуется на условиях лицензии Creative Commons Attribution 4.0 International (https://creativecommons.org/licenses/by/4.0/deed.ru)

\section{Образец для цитирования}

З ар уб и н А. Н. Нелокальная краевая задача Трикоми для дифференциально-разностного уравнения смешанного типа // Вестн. Сам. гос. техн. ун-та. Сер. Физ.-мат. науки, 2021. T. 25, № 1. C. 35-50. https://doi.org/10.14498/vsgtu1835.

\section{Сведения об авторе}

Александр Николаевич Зарубин (1) https://orcid.org/0000-0002-0611-5752

доктор физико-математических наук, профессор; заведующий кафедрой; каф. математического анализа и дифференциальных уравнений; e-mail:aleks_zarubin@mail.ru; matdiff@yandex.ru 


$$
\begin{aligned}
& \sum_{k=-1}^{1} \sum_{n=-1}^{1} H\left((-1)^{(k-1) / 2} k(y+(k-1) h / 2)\right) \times \\
& \times\left[(\operatorname{sgn} y) b_{n+1, k+1} U_{y y}(x+n \tau, y+k h)+a_{n+1, k+1} U_{x x}(x-n \tau, y+k h)\right]=0
\end{aligned}
$$

в области $D=D^{+} \cup D^{-} \cup I^{0}$, где

$$
D^{+}=\{(x, y): 0<x<3 \tau, 0<y<2 h\}=\bigcup_{k=0}^{2}\left(\bigcup_{j=0}^{1} D_{k j}^{+}\right),
$$

и

$$
D^{-}=\bigcup_{k=0}^{2} D_{k 0}^{-}=\bigcup_{k=0}^{2}\left(\bigcup_{j=0}^{1} D_{k 0}^{\gamma_{j 0}}\right)
$$

- эллиптическая и гиперболическая части области $D$, причем

$$
D_{k j}^{+}=\{(x, y): k \tau<x<(k+1) \tau, j h<y<(j+1) h\}, \quad k=-\overline{1,3}, \quad j=0,1,2 ;
$$

$0<\tau, h, a_{n+1, k+1}, b_{n+1, k+1} \equiv$ const; $H(\zeta)$ - функция Хевисайда; $D_{k 0}^{\gamma_{j 0}}=$ $=\left\{(x, y):-y \gamma_{j 0}+k \tau<x<y \gamma_{j 0}+(k+1) \tau ;-\tau / 2 \gamma_{j 0}<y<0\right\}, k=-\overline{1,3}$, $j=0,1 ; \gamma_{j k}^{2}-$ собственные значения матрицы коэффициентов уравнения (1). Пусть $D_{k}=D_{k 0}^{-} \bigcup_{j=0}^{1} D_{k j}^{+} \bigcup_{l=0}^{1} I_{k}^{l}, k=-\overline{1,3}$, где

$$
I^{l}=\bigcup_{k=0}^{2} I_{k}^{l}, \quad I_{k}^{l}=\{(x, y): k \tau<x<(k+1) \tau, y=l h\}, \quad l=0,1,
$$

a

$$
J=\bigcup_{k=0}^{1} J_{k}, \quad J_{k}=\{(x, y): x=(k+1) \tau, 0<y<2 h\} .
$$

Тогда $D=\bigcup_{k=0}^{2} D_{k} \bigcup_{j=0}^{1} J_{j}$.

1. Постановка задачи. Редукция. Дифференциально-разностное уравнение смешанного типа (1) запишем в виде

$$
\begin{aligned}
& \sum_{k=-1}^{1} H\left((-1)^{(k-1) / 2} k(y+(k-1) h / 2)\right) \times \\
& \times\left\{( \operatorname { s g n } y ) \left[b_{0(k+1)} U_{y y}(x-\tau, y+k h)+b_{1(k+1)} U_{y y}(x, y+k h)+\right.\right. \\
& \left.+b_{2(k+1)} U_{y y}(x+\tau, y+k h)\right]+ \\
& +\left[a_{0(k+1)} U_{x x}(x+\tau, y+k h)+a_{1(k+1)} U_{x x}(x, y+k h)+\right. \\
& \left.\left.+a_{2(k+1)} U_{x x}(x-\tau, y+k h)\right]\right\}=0, \quad(x, y) \in D .
\end{aligned}
$$

ЗАДАча Т. В области $D=\bigcup_{k=0}^{2} D_{k} \bigcup J$ найти решение $U(x, y) \in C(\bar{D}) \bigcap$ $\cap C^{2}\left(D \backslash\left(J \bigcup_{l=0}^{1} I^{l}\right)\right)$ уравнения (2), удовлетворяющее следующим условиям:

$$
U(x, y)=r(x, y), \quad(x, y) \in \bar{D}_{-1}
$$




$$
\begin{aligned}
& U(x, y)=\rho(x, y), \quad(x, y) \in \bar{D}_{3} \\
& U(x, y)=\delta(x, y), \quad(x, y)=\overline{\bigcup_{k=0}^{2} D_{k 2}^{+}} ; \\
& U\left(x,(k \tau-x) / \gamma_{j 0}\right)=\psi_{k j}(x), k \tau \leqslant x \leqslant(2 k+1) \tau / 2, j=0,1, k=0,1,2,
\end{aligned}
$$

и условиям сопряжения

$$
\begin{gathered}
U(x, 0-)=U(x, 0+)=\omega(x), \quad 0 \leqslant x \leqslant 3 \tau \\
U_{y}(x, 0-)=U_{y}(x, 0+)=\nu(x), \quad 0<x<3 \tau, x \neq \tau, 2 \tau .
\end{gathered}
$$

Причем

$$
\begin{gathered}
\psi_{0 j}(0)=r(0,0), \quad r(0,2 h)=\delta(0,2 h), \quad \rho(3 \tau, 2 h)=\delta(3 \tau, 2 h), \\
r(0, y)=\rho(3 \tau, y), \quad 0 \leqslant y \leqslant 2 h ;
\end{gathered}
$$

$\gamma_{j k}^{2}$ - собственные значения матрищъ коэфбищиентов уравнения $(2), j=0,1$, $k=0,1,2 ; r(x, y), \rho(x, y), \delta(x, y), \psi_{k j}(x)$ - заданные непрерывные достаточно гладкие функиии; $\omega(x), \nu(x)$ - бункиии, подлежащие определению в прочессе решения задачи $T$.

Tеорема 1. Если

$$
\begin{gathered}
r(x, y) \in C\left(\bar{D}_{-1}\right) \cap C^{4}\left(D_{-1}\right) ; \quad \rho(x, y) \in C\left(\bar{D}_{2}\right) \cap C^{4}\left(D_{2}\right) ; \\
\delta(x, y) \in C\left(\overline{\left(\bigcup_{k=0}^{2} D_{k 2}^{+}\right)} \cap C^{4}\left(\bigcup_{k=0}^{2} D_{k 2}^{+}\right) ;\right.
\end{gathered}
$$

$\psi_{k j}(x) \in C[k \tau,(2 k+1) \tau / 2] \bigcap C^{2}(k \tau,(2 k+1) \tau / 2), \quad k=0,1,2, j=0,1 ;$ $r(0, y)=\rho(3 \tau, y), 0 \leqslant y \leqslant 2 h ; r(0,0)=\psi_{0 j}(0) ; r(0,2 h)=\delta(0,2 h) ; \rho(3 \tau, 2 h)=$ $=\delta(3 \tau, 2 h)$, то существует единственное решение задачи $T$.

Для до ка з $а$ m e л в $c$ в в а теоремы произведем редукцию опережающе-запаздывающего уравнения смешанного типа (2) сначала к системе трех уравнений смешанного типа без отклонений по переменной $x$, а затем к системе шести уравнений смешанного типа без отклонений и по аргументу $y$.

В терминах функций

$$
U_{k}(x, y)=U(x, y), \quad(x, y) \in D_{k}, \quad k=-\overline{1,3},
$$

учитывая $(3),(4)$, переводя $D_{k}$ заменой $x$ на $x+k \tau(k=0,1,2)$ в $D_{0}$ и используя вектор

$$
\bar{U}(x, y)=\left(U_{0}(x, y), U_{1}(x+\tau, y), U_{2}(x+2 \tau, y)\right)^{\top}, \quad(x, y) \in D_{0},
$$

запишем уравнение $(2)$ в областях $D_{k}(k=0,1,2)$ в форме

$$
\begin{aligned}
\sum_{k=-1}^{1} H\left((-1)^{(k-1) / 2} k(y\right. & +(k-1) h / 2)) \times \\
& \times\left\{(\operatorname{sgn} y) B_{k} \bar{U}_{y y}(x, y+k h)+A_{k} \bar{U}_{x x}(x, y+k h)\right\}=
\end{aligned}
$$




$$
\begin{aligned}
& =-\sum_{k=-1}^{1} H\left((-1)^{(k-1) / 2} k(y+(k-1) h / 2)\right) \times \\
& \quad \times\left\{(\operatorname{sgn} y) B_{k}^{0} \bar{m}_{y y}(x, y+k h)+A_{k}^{0} \bar{m}_{x x}(x, y+k h)\right\}, \quad(x, y) \in D_{0},
\end{aligned}
$$

где

$$
\begin{gathered}
B_{k}=\left(\begin{array}{ccc}
b_{1(k+1)} & b_{2(k+1)} & 0 \\
b_{0(k+1)} & b_{1(k+1)} & b_{2(k+1)} \\
0 & b_{0(k+1)} & b_{1(k+1)}
\end{array}\right), \quad A_{k}=\left(\begin{array}{ccc}
a_{1(k+1)} & a_{0(k+1)} & 0 \\
a_{2(k+1)} & a_{1(k+1)} & a_{0(k+1)} \\
0 & a_{2(k+1)} & a_{1(k+1)}
\end{array}\right), \\
\bar{m}(x, y+k h)=(r(x-\tau, y+k h), 0, \rho(x+3 \tau, y+k h))^{\top},
\end{gathered}
$$

причем

$$
B_{k}^{0}=\left(\begin{array}{ccc}
b_{0(k+1)} & 0 & 0 \\
0 & 0 & 0 \\
0 & 0 & b_{2(k+1)}
\end{array}\right), \quad A_{k}^{0}=\left(\begin{array}{ccc}
a_{2(k+1)} & 0 & 0 \\
0 & 0 & 0 \\
0 & 0 & a_{0(k+1)}
\end{array}\right) .
$$

Когда $b_{2(k+1)}=b_{0(k+1)}, b_{1(k+1)}>\sqrt{2} b_{0(k+1)}>0, a_{2(k+1)}=a_{0(k+1)}, a_{1(k+1)}>$ $>\sqrt{2} a_{0(k+1)}>0$, матрицы $B_{k}, A_{k}$ симметричны и попарно коммутативны. Поэтому [6] существует невырожденная матрица $T$ такая, что

$$
T^{-1} B_{k} T=\Lambda_{B_{k}}=\left(\begin{array}{ccc}
\alpha_{0(k+1)}^{2} & 0 & 0 \\
0 & \alpha_{1(k+1)}^{2} & 0 \\
0 & 0 & \alpha_{2(k+1)}^{2}
\end{array}\right) \text {, }
$$

где $\alpha_{j(k+1)}^{2}=b_{1(k+1)}-(-1)^{j} j \sqrt{2} b_{0(k+1)} 2^{1-j}(j=\overline{0,2} ; k=-\overline{1,1})$ - собственные значения матрицы $B_{k}$;

$$
T^{-1} A_{k} T=\Lambda_{A_{k}}=\left(\begin{array}{ccc}
\beta_{0(k+1)}^{2} & 0 & 0 \\
0 & \beta_{1(k+1)}^{2} & 0 \\
0 & 0 & \beta_{2(k+1)}^{2}
\end{array}\right)
$$

где $\beta_{j(k+1)}^{2}=a_{1(k+1)}-(-1)^{j} j \sqrt{2} a_{0(k+1)} 2^{1-j}(j=\overline{0,2} ; k=-\overline{1,1})-$ собственные значения матрицы $A_{k}$. При этом

$$
T=\left(\begin{array}{ccc}
1 & 1 & 1 \\
0 & \sqrt{2} & -\sqrt{2} \\
-1 & 1 & 1
\end{array}\right), \quad T^{-1}=\frac{1}{4}\left(\begin{array}{ccc}
2 & 0 & -2 \\
1 & \sqrt{2} & 1 \\
1 & -\sqrt{2} & 1
\end{array}\right)=\frac{1}{4}\left(\begin{array}{l}
\bar{P}_{0} \\
\bar{P}_{1} \\
\bar{P}_{2}
\end{array}\right)
$$

Умножая слева матричное уравнение (9) на $T^{-1}$ и учитывая соотношения

$$
\begin{gathered}
T^{-1} B_{k}=\Lambda_{B_{k}} T^{-1}, \quad T^{-1} A_{k}=\Lambda_{A_{k}} T^{-1} \\
T^{-1} B_{k}^{0} \bar{m}=b_{0(k+1)} T^{-1} \bar{m}, \quad T^{-1} A_{k}^{0} \bar{m}=a_{0(k+1)} T^{-1} \bar{m}
\end{gathered}
$$


после преобразований получим три отдельных опережающе-запаздывающих только по переменной $у$ уравнения смешанного типа:

$$
\begin{aligned}
& \sum_{k=-1}^{1} H\left((-1)^{(k-1) / 2} k(y+(k-1) h / 2)\right) \times \\
& \quad \times\left\{(\operatorname{sgn} y) \alpha_{j(k+1)}^{2}\left\langle\bar{P}_{j}, \bar{U}(x, y+k h)\right\rangle_{y y}+\beta_{j(k+1)}^{2}\left\langle\bar{P}_{j}, \bar{U}(x, y+k h)\right\rangle_{x x}\right\}= \\
& =-\sum_{k=-1}^{1} H\left((-1)^{(k-1) / 2} k(y+(k-1) h / 2)\right) \times \\
& \times\left\{(\operatorname{sgn} y) b_{0(k+1)}\left\langle\bar{P}_{j}, \bar{m}(x, y+k h)\right\rangle_{y y}+\quad(x, y) \in D_{0},\right. \\
& \left.\quad+a_{0(k+1)}\left\langle\bar{P}_{j}, \bar{m}(x, y+k h)\right\rangle_{x x}\right\}, \quad(1)
\end{aligned}
$$

где $\left\langle\bar{P}_{j}, \bar{U}\right\rangle,\left\langle\bar{P}_{j}, \bar{m}\right\rangle$ - скалярное произведение векторов, $j=0,1,2$; область $D_{0}=D_{00}^{-} \cup D_{00}^{+} \cup D_{01}^{+}$.

Уравнение $(10)$ в области $D_{00}^{-}$является гиперболическим без отклонений аргументов $x$ и $y$ :

$$
\begin{aligned}
& -\alpha_{j 1}^{2}\left\langle\bar{P}_{j}, \bar{U}(x, y)\right\rangle_{y y}+\beta_{j 1}^{2}\left\langle\bar{P}_{j}, \bar{U}(x, y)\right\rangle_{x x}= \\
& \quad=b_{01}\left\langle\bar{P}_{j}, \bar{m}(x, y)\right\rangle_{y y}-a_{01}\left\langle\bar{P}_{j}, \bar{m}(x, y)\right\rangle_{x x}, \quad(x, y) \in D_{00}^{-}, \quad j=0,1,2,
\end{aligned}
$$

причем, так же как в (8), (7), будем считать

$$
\begin{aligned}
& \bar{U}(x, y)=\left(U_{00}(x, y), U_{10}(x+\tau, y), U_{20}(x+2 \tau, y)\right)^{\top}, \\
& \bar{U}(x, y+h)=\left(U_{01}(x, y+h), U_{11}(x+\tau, y+h), U_{21}(x+2 \tau, y+h)\right)^{\top}
\end{aligned}
$$

при $(x, y) \in D_{00}$. Здесь

$$
U_{k j}(x, y)=U_{k}(x, y)=U(x, y), \quad(x, y) \in D_{k j}, \quad k=0,1,2, \quad j=0,1 .
$$

В терминах функций $(13),(12)$, учитывая $(5)$ и переводя $D_{0 k}^{+}$заменой $y$ на $y+k h(k=0,1)$ в $D_{00}^{+}$, запишем уравнение $(10)$ в областях $D_{0 k}^{+}(k=0,1)$ в виде системы

$$
\begin{aligned}
& M\left(\begin{array}{c}
\left\langle\bar{P}_{j}, \bar{U}(x, y)\right\rangle \\
\left\langle\bar{P}_{j}, \bar{U}(x, y+h)\right\rangle
\end{array}\right)_{y y}+N\left(\begin{array}{c}
\left\langle\bar{P}_{j}, \bar{U}(x, y)\right\rangle \\
\left\langle\bar{P}_{j}, \bar{U}(x, y+h)\right\rangle
\end{array}\right)_{x x}= \\
& =-M_{0}\left(\begin{array}{c}
\left\langle\bar{P}_{j}, \bar{m}(x, y)\right\rangle \\
\left\langle\bar{P}_{j}, \bar{m}(x, y+h)\right\rangle
\end{array}\right)_{y y}+N_{0}\left(\begin{array}{c}
\left\langle\bar{P}_{j}, \bar{m}(x, y)\right\rangle \\
\left\langle\bar{P}_{j}, \bar{m}(x, y+h)\right\rangle
\end{array}\right)_{x x}- \\
& -H(y) \alpha_{j 2}^{2}\left(\begin{array}{c}
0 \\
\left\langle\bar{P}_{j}, \bar{U}^{\delta}(x, y+2 h)\right\rangle
\end{array}\right)_{y y}-H(y) \beta_{j 2}^{2}\left(\begin{array}{c}
0 \\
\left\langle\bar{P}_{j}, \bar{U}^{\delta}(x, y+2 h)\right\rangle
\end{array}\right)_{x x}- \\
& -H(y) b_{02}\left(\left\langle\bar{P}_{j}, \bar{m}(x, y+2 h)\right\rangle\right)_{y y}-H(y) a_{02}\left(\begin{array}{c}
0 \\
\left\langle\bar{P}_{j}, \bar{m}(x, y+2 h)\right\rangle
\end{array}\right)_{x x}, \\
& (x, y) \in D_{00}^{+}, \quad j=0,1,2 \text {, }
\end{aligned}
$$

где, согласно (12), (13), (5), 
$\bar{U}^{\delta}(x, y+2 h)=(\delta(x, y+2 h), \delta(x+\tau, y+2 h), \delta(x+2 \tau, y+2 h))^{\top}, \quad(x, y) \in D_{00}^{+}$, причем

$$
M=\left(\begin{array}{cc}
\alpha_{j 1}^{2} & H(y) \alpha_{j 2}^{2} \\
H(y) \alpha_{j 0}^{2} & \alpha_{j 1}^{2}
\end{array}\right), \quad N=\left(\begin{array}{cc}
\beta_{j 1}^{2} & H(y) \beta_{j 2}^{2} \\
H(y) \beta_{j 0}^{2} & \beta_{j 1}^{2}
\end{array}\right),
$$

a

$$
M_{0}=\left(\begin{array}{cc}
b_{01} & H(y) b_{02} \\
H(y) b_{00} & b_{01}
\end{array}\right), \quad N_{0}=\left(\begin{array}{cc}
a_{01} & H(y) a_{02} \\
H(y) a_{00} & a_{01}
\end{array}\right) .
$$

При $\alpha_{j 2}^{2}=\alpha_{j 0}^{2}, \beta_{j 2}^{2}=\beta_{j 1}^{2}$ (в этом случае $b_{02}=b_{00}, a_{02}=a_{00}$ ) матрицы $M$ и $N$ (матрицы $M_{0}$ и $N_{0}$ ) симметричны и попарно коммутативны. Поэтому [6] существует невырожденная матрица $Q$ такая, что

$$
Q^{-1} M Q=\Lambda_{M}=\left(\begin{array}{cc}
\alpha_{j 1}^{2}+H(y) \alpha_{j 0}^{2} & 0 \\
0 & \alpha_{j 1}^{2}-H(y) \alpha_{j 0}^{2}
\end{array}\right),
$$

где $\alpha_{j 1}^{2}+(-1)^{k} \alpha_{j 0}^{2}(k=0,1)$ - собственные значения матрицы $M$;

$$
Q^{-1} N Q=\Lambda_{N}=\left(\begin{array}{cc}
\beta_{j 1}^{2}+H(y) \beta_{j 0}^{2} & 0 \\
0 & \beta_{j 1}^{2}-H(y) \beta_{j 0}^{2}
\end{array}\right),
$$

где $\beta_{j 1}^{2}+(-1)^{k} \beta_{j 0}^{2}(k=0,1)$ - собственные значения матрицы $N$;

$$
Q^{-1} M_{0} Q=\Lambda_{M_{0}}=\left(\begin{array}{cc}
b_{01}+H(y) b_{00} & 0 \\
0 & b_{01}-H(y) b_{00}
\end{array}\right),
$$

где $b_{01}+(-1)^{k} b_{00}(k=0,1)$ - собственные значения матрицы $M_{0}$;

$$
Q^{-1} N_{0} Q=\Lambda_{N_{0}}=\left(\begin{array}{cc}
a_{01}+H(y) a_{00} & 0 \\
0 & a_{01}-H(y) a_{00}
\end{array}\right),
$$

где $a_{01}+(-1)^{k} a_{00}(k=0,1)$ - собственные значения матрицы $N_{0}$. При этом

$$
Q=\left(\begin{array}{cc}
1 & 1 \\
1 & -1
\end{array}\right), \quad Q^{-1}=\frac{1}{2} Q .
$$

Умножая слева матричное уравнение (14) на $Q^{-1}$ и учитывая соотношения

$$
\begin{array}{cl}
Q^{-1} M=\Lambda_{M} Q^{-1}, & Q^{-1} N=\Lambda_{N} Q^{-1}, \\
Q^{-1} M_{0}=\Lambda_{M_{0}} Q^{-1}, & Q^{-1} N_{0}=\Lambda_{N_{0}} Q^{-1},
\end{array}
$$

после преобразований получим шесть отдельных уравнений эллиптического типа без отклонения аргументов:

$$
\begin{aligned}
\left(\alpha_{j 1}^{2}+\right. & \left.(-1)^{k} H(y) \alpha_{j 0}^{2}\right)\left(\left\langle\bar{P}_{j}, \bar{U}(x, y)+(-1)^{k} \bar{U}(x, y+h)\right\rangle\right)_{y y}+ \\
& +\left(\beta_{j 1}^{2}+(-1)^{k} H(y) \beta_{j 0}^{2}\right)\left(\left\langle\bar{P}_{j}, \bar{U}(x, y)+(-1)^{k} \bar{U}(x, y+h)\right\rangle\right)_{x x}= \\
= & -\left(b_{01}+(-1)^{k} H(y) b_{00}\right)\left(\left\langle\bar{P}_{j}, \bar{m}(x, y)+(-1)^{k} \bar{m}(x, y+h)\right\rangle\right)_{y y}- \\
& \left.\quad-\left(a_{01}+(-1)^{k} H(y) a_{00}\right\rangle\right)\left(\left\langle\bar{P}_{j}, \bar{m}(x, y)+(-1)^{k} \bar{m}(x, y+h)\right\rangle\right)_{x x}-
\end{aligned}
$$




$$
\begin{array}{r}
-H(y) \alpha_{j 2}^{2}(-1)^{k}\left(\left\langle\bar{P}_{j}, \bar{U}^{\delta}(x, y+2 h)\right\rangle\right)_{y y}-H(y) \beta_{j 2}^{2}(-1)^{k}\left(\left\langle\bar{P}_{j}, \bar{U}^{\delta}(x, y+2 h)\right\rangle\right)_{x x}- \\
-H(y) b_{02}(-1)^{k}\left(\left\langle\bar{P}_{j}, \bar{m}(x, y+2 h)\right\rangle\right)_{y y}-H(y) a_{02}(-1)^{k}\left(\left\langle\bar{P}_{j}, \bar{m}(x, y+2 h)\right\rangle\right)_{x x} \\
(x, y) \in D_{00}^{+}, \quad j=0,1,2, \quad k=0,1 .
\end{array}
$$

Таким образом, опережающе-запаздывающее уравнение смешанного типа (2) в силу (11), (15) приведено к системе шести уравнений смешанного типа без отклонений аргументов:

$$
(\operatorname{sgn} y) q_{j k y y}(x, y)+\gamma_{j k}^{2} q_{j k x x}(x, y)=n_{j k}(x, y), \quad(x, y) \in D_{00},
$$

где

$$
\begin{gathered}
q_{j k}(x, y)=\frac{1}{8}\left\langle\bar{P}_{j}, \bar{U}(x, y)+(-1)^{k} H(y) \bar{U}(x, y+h)\right\rangle \\
\gamma_{j k}^{2}=\frac{\beta_{j 1}^{2}+(-1)^{k} H(y) \beta_{j 0}^{2}}{\alpha_{j 1}^{2}+(-1)^{k} H(y) \alpha_{j 0}^{2}} \\
n_{j k}(x, y)=-\frac{1}{8\left[\alpha_{j 1}^{2}+(-1)^{k} H(y) \alpha_{j 0}^{2}\right]}\left\{\left\langle\bar{P}_{j},\left[(\operatorname{sgn} y)\left(b_{01}+(-1)^{k} H(y) b_{00}\right) \frac{\partial^{2}}{\partial y^{2}}+\right.\right.\right. \\
\left.\left.+\left(a_{01}+(-1)^{k} H(y) a_{00}\right) \frac{\partial^{2}}{\partial x^{2}}\right]\left(\bar{m}(x, y)+(-1)^{k} H(y) \bar{m}(x, y+h)\right)\right\rangle+ \\
+H(y)(-1)^{k}\left\langle\bar{P}_{j},\left[(\operatorname{sgn} y) \alpha_{j 2}^{2} \frac{\partial^{2}}{\partial y^{2}}+\beta_{j 2}^{2} \frac{\partial^{2}}{\partial x^{2}}\right] \bar{U} \delta(x, y+2 h)\right\rangle+ \\
\left.+H(y)(-1)^{k}\left\langle\bar{P}_{j},\left[(\operatorname{sgn} y) b_{02} \frac{\partial^{2}}{\partial y^{2}}+a_{02} \frac{\partial^{2}}{\partial x^{2}}\right] \bar{m}(x, y+2 h)\right\rangle\right\} .
\end{gathered}
$$

Множество решений $q_{j k}(x, y),(x, y) \in D_{00}(j=0,1,2, k=0,1)$ шести неоднородных уравнений смешанного типа Лаврентьева-Бицадзе (16) содержит все решения $U(x, y)=U_{j k}(x, y),(x, y) \in D_{j k}(j=0,1,2, k=0,1)$ опережающе-запаздывающего уравнения смешанного типа (2), которые в силу (12) можно выделить из системы (17) в виде

$$
\begin{gathered}
\left(\begin{array}{c}
U_{0 l}(x, y+l h) \\
U_{1 l}(x+\tau, y+l h) \\
U_{2 l}(x+2 \tau, y+l h)
\end{array}\right)=\bar{U}(x, y+l h)= \\
=T\left(\begin{array}{l}
q_{00}(x, y)+(-1)^{l} q_{01}(x, y) \\
q_{10}(x, y)+(-1)^{l} q_{11}(x, y) \\
q_{20}(x, y)+(-1)^{l} q_{21}(x, y)
\end{array}\right), \quad(x, y) \in D_{00}, l=0,1 .
\end{gathered}
$$

Таким образом, поставленная задача Т для опережающе-запаздывающего уравнения смешанного типа $(2)$ в области $D=D^{+} \bigcup D^{-} \bigcup I$ относительно искомой функции $U(x, y)$ редуцирована к шести задачам Трикоми для шести уравнений смешанного типа (16) без отклонений в области $D_{00}=$ $=D_{00}^{+} \bigcup D_{00}^{-} \bigcup I_{0}$ относительно функций $q_{j k}(x, y)$ вида $(17)$. 
ЗАДАча $T_{j k}$. B области $D_{00}=D_{00}^{+} \bigcup D_{00}^{-} \bigcup I_{0}$ найти решение $q_{j k}(x, y) \in$ $\in C\left(\bar{D}_{00}\right) \bigcap C^{2}\left(D_{00} \backslash I_{0}\right)$ уравнения $(16)$, удовлетворяющее условиям

$$
\begin{gathered}
q_{j k}(0, y)=q_{j k}(\tau, y)=\bar{r}_{j k}(y) \equiv \frac{1}{8}\left\langle\bar{P}_{j}, \bar{U}(0, y)+(-1)^{k} \bar{U}(0, y+h)\right\rangle, \quad 0 \leqslant y \leqslant h \\
q_{j k}(x, h)=\bar{\delta}_{j k}(x) \equiv \frac{1}{8}\left\langle\bar{P}_{j}, \bar{U}(x, h)+(-1)^{k} \bar{U}(x, 2 h)\right\rangle, \quad 0 \leqslant x \leqslant \tau \\
q_{j k}\left(x,-x / \gamma_{j k}\right)=\bar{\psi}_{j k}(x) \equiv \frac{1}{8}\left\langle\bar{P}_{j}, \bar{U}\left(x,-x / \gamma_{j k}\right)\right\rangle, \quad 0 \leqslant x \leqslant \tau / 2
\end{gathered}
$$

условиям сопряжения

$$
\begin{gathered}
q_{j k}(x, 0-)=q_{j k}(x, 0+)=\bar{\omega}_{j k}(x) \equiv \frac{1}{8}\left\langle\bar{P}_{j}, \bar{U}(x, 0)\right\rangle, \quad 0 \leqslant x \leqslant \tau \\
q_{j k y}(x, 0-)=q_{j k y}(x, 0+)=\bar{\nu}_{j k}(x) \equiv \frac{1}{8}\left\langle\bar{P}_{j}, \bar{U}_{y}(x, 0)\right\rangle, \quad 0<x<\tau
\end{gathered}
$$

причем

$$
\bar{r}_{j k}(0)=\bar{\psi}_{j k}(0), \quad \bar{r}_{j k}(h)=\bar{\delta}_{j k}(0), \quad \bar{r}_{j k}(h)=\bar{\delta}_{j k}(\tau),
$$

где $\bar{r}_{j k}(y), \bar{\delta}_{j k}(x), \bar{\psi}_{j k}(x)$ - заданные непрерывные достаточно гладкие функиии; $\bar{\omega}_{j k}(x), \bar{\nu}_{j k}(x)$ - функиии, подлежсашие определению в процессе решения задачи $T_{j k}$. Здесь и далее $j=0,1,2, k=0,1$.

2. Однозначная разрешимость задачи $T$. Единственность решения задачи $T$ для опережающе-запаздывающего уравнения смешанного типа (2) в области $D=D^{+} \cup D^{-} \bigcup I$ следует из того, что однородная задача $T$ имеет тривиальное решение $U(x, y) \equiv 0$ в $\bar{D}$ в смысле ее эквивалентности, согласно $(1),(18)$, тривиальному решению $q_{j k}(x, y) \equiv 0$ в $\bar{D}_{00}=\bar{D}_{00}^{+} \bigcup \bar{D}_{00}^{-} \bigcup I_{0}$ однородной задаче $T_{j k}$ для однородного уравнения (16) при однородных условиях (19)-(21).

Доказ ательство этого факта основано на установлении знакоопределенности интеграла

$$
\beta_{j k}=\int_{0}^{\tau} \bar{\omega}_{j k}(x) \bar{\nu}_{j k}(x) d x
$$

Лемма 1. Если $q_{j k}(x, y)$-решение однородного уравнения (16) в области $\bar{D}_{00}^{+}$из класса $C\left(\bar{D}_{00}^{+}\right) \bigcap C^{2}\left(D_{00}^{+}\right)$, обращающееся в нуль при $x=0, x=\tau$ $(0 \leqslant y \leqslant h)$ u $y=h(0 \leqslant x \leqslant \tau)$, mo

$$
\begin{gathered}
\beta_{j k} \leqslant 0 \\
\beta_{j k}+\iint_{D_{00}^{+}}\left[q_{j k y}^{2}(x, y)+\gamma_{j k}^{2} q_{j k x}^{2}(x, y)\right] d x d y=0 .
\end{gathered}
$$

ЛЕмма 2. Eсли $q_{j k}(x, y) \in C\left(\bar{D}_{00}^{-}\right) \bigcap C^{2}\left(D_{00}^{-}\right)$- решение однородного уравнения (16) в области $D_{00}^{-}$, обращаюеееся в нуль на характеристике $x=-\gamma_{j k}$ y $(0 \leqslant x \leqslant \tau / 2)$, mo

$$
\beta_{j k} \geqslant 0 .
$$


Дока зательст в о лемм 1, 2 можно провести аналогично [7,8].

Из неравенств $(24),(26)$ следует $\beta_{j k}=0$, поэтому из равенства (25) получим положительно определенную форму, равную нулю, и, значит, $q_{j k x}(x, y) \equiv 0$, $q_{j k y}(x, y) \equiv 0$, т. е. $q_{j k}(x, y) \equiv$ const в $D_{00}^{+}$. Однородность граничных условий в $D_{00}^{+}$и $q_{j k}(x, y) \in C\left(\bar{D}_{00}^{+}\right)$позволяют утверждать, что $q_{j k}(x, y) \equiv 0$ в $\bar{D}_{00}^{+}$и, в частности, $q_{j k}(x, 0)=0,0 \leqslant x \leqslant \tau$. Последнее равенство в совокупности с однородным условием (21) обеспечивает тривиальность решений $q_{j k}(x, y) \equiv 0$ первой задачи Дарбу в $\bar{D}_{00}^{-}$. Из полученной тривиальности решений $q_{j k}(x, y)$ в $\bar{D}_{00}^{+}$и $\bar{D}_{00}^{-}$следует тривиальность $q_{j k}(x, y) \equiv 0$ в $\bar{D}_{00}$.

Таким образом, единственность решения задачи $T_{j k}$ для уравнения (16) и граничных условий $(19)-(21)$ в области $\bar{D}_{00}$ доказана.

Тривиальность решения однородной задачи $T$ для опережающе-запаздывающего уравнения смешанного типа (2) и однородных граничных условий $(3)-(6)$ в области $\bar{D}$ следует из $q_{j k}(x, y) \equiv 0$ в $\bar{D}_{00}$ и равенств $(18),(12),(13)$ : $U(x, y)=U_{j k}(x, y) \equiv 0,(x, y) \in \bar{D}_{j k}$. Это означает единственность решения задачи $T$ для уравнения (2) при граничных условиях (3)-(6) в области $\bar{D}$.

Дока з ат ель ст в о существования решения $U(x, y)$ задачи Т в области $\bar{D}$ для опережающе-запаздывающего уравнения смешанного типа (2) основано на решениях задач $T_{j k}$ в области эллиптичности $D_{00}^{+}$и гиперболичности $D_{00}^{-}$для уравнения $(16)$.

ЗАДАчА НЕЙМАНА-ДиРИХЛЕ. В области $D_{00}^{+}$найти решение $q_{j k}^{+}(x, y) \in$ $\in C\left(\bar{D}_{00}^{+}\right) \bigcap C^{2}\left(D_{00}^{+}\right)$уравнения $(16):$

$$
q_{j k y y}^{+}(x, y)+\gamma_{j k}^{2} q_{j k x x}^{+}(x, y)=n_{j k}^{+}(x, y), \quad(x, y) \in D_{00}^{+},
$$

где $n^{+}(x, y)=n_{j k}(x)$, удовлетворяющее условиям (19), (20), (23).

ЗАДАЧА ДАРБУ. В области $D_{00}^{-}$найти решение $q_{j k}^{-}(x, y) \in C\left(\bar{D}_{00}^{-}\right) \cap C^{2}\left(D_{00}^{-}\right)$ уравнения (16):

$$
\begin{aligned}
q_{j k y y}^{-}-\gamma_{j k}^{2} & q_{j k x x}^{-}(x, y)=n_{j k}^{-}(x, y) \equiv \\
& \equiv \frac{1}{8 \alpha_{j 1}^{2}}\left\langle\bar{P}_{j},\left(b_{01} \partial^{2} / \partial y^{2}-a_{01} \partial^{2} / \partial x^{2}\right) \bar{m}(x, y)\right\rangle, \quad(x, y) \in D_{00}^{-},
\end{aligned}
$$

удовлетворяющее условиям (21), (23).

Вопрос существования решения $q_{j k}(x, y)$ задачи $T_{j k}$ для уравнения $(16)$ в области $D_{00}=D_{00}^{+} \bigcup D_{00}^{-} \cup I_{0}$ связан с разрешимостью полного [9] сингулярного интегрального уравнения относительно $\bar{\nu}_{j k}(x), 0<x<\tau$, которое будет получено из функциональных соотношений между $\bar{\omega}_{j k}(x)$ и $\bar{\nu}_{j k}(x)$, привнесенных на $y=0,0<x<\tau$ решениями задачи Неймана-Дирихле из $D_{00}^{+}$ и задачи Дарбу из $D_{00}^{-}$.

Лемма 3. Если имеют место включения

$$
\bar{r}_{j k}(y) \in C[0, h] \bigcap C^{2}(0, h), \quad \bar{\delta}_{j k}(x) \in C[0, \tau] \bigcap C^{2}(0, \tau), \quad \bar{\nu}_{j k}(x) \in C^{1}(0, \tau),
$$


то существует единственное решение задачи Неймана-Дирихле $q_{j k}^{+}(x, y) \in$ $\in C\left(\bar{D}_{00}^{+}\right) \bigcap C^{2}\left(D_{00}^{+}\right)$, которое имеет вид

$$
\begin{aligned}
q_{j k}^{+}(x, y)=\int_{0}^{\tau} \bar{\delta}_{j k}(t) \frac{\partial}{\partial y} G_{j k}(x, t ; 0, y) d t- & \\
-\int_{0}^{\tau} \bar{\nu}_{j k}(t) G_{j k}(x, t ; 0, h-y) d t & -\int_{0}^{h} d \zeta \int_{0}^{\tau} n_{j k}^{+}(t, \zeta) \Gamma_{j k}(x, t ; \zeta, y) d t+ \\
& +\int_{0}^{h} d \zeta \int_{0}^{\tau} \bar{r}_{j k}(\zeta) \frac{\partial^{2}}{\partial y^{2}} \Gamma_{j k}(x, t ; \zeta, y) d t
\end{aligned}
$$

zde

$$
\Gamma_{j k}(x, y ; \zeta, y)= \begin{cases}G_{j k}(x, t ; \zeta, h-y), & y>\zeta \\ G_{j k}(x, t ; y, h-\zeta), & \zeta>y\end{cases}
$$

причем

$$
G_{j k}(x, t ; r, z)=\frac{2}{\tau} \sum_{m=1}^{+\infty} \frac{\operatorname{sh}\left(\gamma_{j k} \lambda_{m} z\right) \operatorname{ch}\left(\gamma_{j k} \lambda_{m} r\right)}{\gamma_{j k} \lambda_{m} \operatorname{ch}\left(\gamma_{j k} \lambda_{m} h\right)} \sin \left(\lambda_{m} x\right) \sin \left(\lambda_{m} t\right)
$$

Д о каз а m eль ст в о. Решение задачи Неймана-Дирихле для уравнения (27) в области $D_{00}^{+}$будем искать в форме ряда

$$
q_{j k}^{+}(x, y)=\bar{r}_{j k}(x, y)+\sum_{m=1}^{+\infty} R_{m j k}(y) \sin \left(\lambda_{m} x\right), \quad(x, y) \in \bar{D}_{00}^{+}, \lambda_{m}=m \pi / \tau
$$

в котором функция $R_{m j k}(y)$, удовлетворяющая уравнению

$$
\begin{aligned}
& R_{m j k}^{\prime \prime}(y)-\gamma_{j k}^{2} \lambda_{m}^{2} R_{m j k}(y)=f_{m j k}(y) \equiv \\
& \equiv \frac{2}{\tau} \int_{0}^{\tau}\left[n_{j k}^{+}(\zeta, y)-\bar{r}_{j k}^{\prime \prime}(y)\right] \sin \left(\lambda_{m} \zeta\right) d \zeta, \quad 0<y<h
\end{aligned}
$$

и, в силу (20), (23), условиям

$$
\begin{aligned}
& R_{m j k}(h)=\frac{2}{\tau} \int_{0}^{\tau}\left[\bar{\delta}_{j k}(\zeta)-\bar{r}_{j k}(h)\right] \sin \left(\lambda_{m} \zeta\right) d \zeta, \\
& R_{m j k}^{\prime}(0)=\frac{2}{\tau} \int_{0}^{\tau}\left[\bar{\nu}_{j k}(\zeta)-\bar{r}_{j k}^{\prime}(0)\right] \sin \left(\lambda_{m} \zeta\right) d \zeta,
\end{aligned}
$$

имеет вид

$$
\begin{aligned}
R_{m j k}(y)= & \frac{\operatorname{ch}\left(\gamma_{j k} \lambda_{m} y\right)}{\operatorname{ch}\left(\gamma_{j k} \lambda_{m} h\right)} R_{m j k}(h)-\frac{\operatorname{sh}\left(\gamma_{j k} \lambda_{m}(h-y)\right)}{\gamma_{j k} \lambda_{m} \operatorname{ch}\left(\gamma_{j k} \lambda_{m} h\right)} R_{m j k}^{\prime}(0)- \\
& -\frac{\operatorname{ch}\left(\gamma_{j k} \lambda_{m} y\right)}{\gamma_{j k} \lambda_{m} \operatorname{ch}\left(\gamma_{j k} \lambda_{m} h\right)} \int_{0}^{h} f_{m j k}(\zeta) \operatorname{sh}\left(\gamma_{j k} \lambda_{m}(h-\zeta)\right) d \zeta+ \\
& \quad+\frac{1}{\gamma_{j k} \lambda_{m}} \int_{0}^{y} f_{m j k}(\zeta) \operatorname{sh}\left(\gamma_{j k} \lambda_{m}(y-\zeta)\right) d \zeta, \quad 0 \leqslant y \leqslant h .
\end{aligned}
$$


Подстановка последнего равенства в (30) и необходимые преобразования приводят к искомому решению (29) задачи Неймана-Дирихле (27), (19), $(20),(23)$.

Функииональное соотношение между $\bar{\omega}_{j k}(x)$ и $\bar{\nu}_{j k}(x)$ привнесенное из $D_{00}^{+}$ на $y=0,0 \leqslant x \leqslant \tau$, найдем из решения задачи Неймана-Дирихле (29), полагая $y=0$ и дифференцируя соответствующее выражение:

$$
\begin{aligned}
\bar{\omega}_{j k}^{\prime}(x)=\frac{1}{2 \tau \gamma_{j k}} \int_{0}^{\tau} \bar{\nu}_{j k}(\zeta)[\operatorname{ctg}(\pi(\zeta-x) / 2 \tau)-\operatorname{ctg}(\pi(\zeta+x) / 2 \tau)] d \zeta- \\
-\frac{1}{\tau \gamma_{j k}} \int_{0}^{\tau} \bar{\nu}_{j k}(\zeta) M_{j k}(x, \zeta) d \zeta+\mu_{j k}(x), \quad 0<x<\tau,
\end{aligned}
$$

где

$$
\begin{gathered}
M_{j k}(x, \zeta)=\sum_{n=0}^{+\infty}(-1)^{n}\left[\frac{\sin (\pi(\zeta-x) / \tau)}{\cos (\pi(\zeta-x) / \tau)-\operatorname{ch}\left(2(n+1) \gamma_{j k} \pi h / \tau\right)}+\right. \\
\left.+\frac{\sin (\pi(\zeta+x) / \tau)}{\cos (\pi(\zeta+x) / \tau)-\operatorname{ch}\left(2(n+1) \gamma_{j k} \pi h / \tau\right)}\right], \\
\mu_{j k}(x)=\int_{0}^{\tau} \bar{\delta}_{j k}(t) \frac{\partial}{\partial x}\left[\frac{\partial}{\partial y} G_{j k}(x, t ; 0, y)\right]_{y=0} d t- \\
-\int_{0}^{h} d \zeta \int_{0}^{\tau} n_{j k}^{+}(t, \zeta) \frac{\partial}{\partial x} G_{j k}(x, y ; 0, h-\zeta) d t+ \\
+\int_{0}^{h} d \zeta \int_{0}^{\tau} \bar{r}_{j k}(\zeta) \frac{\partial}{\partial x}\left[\frac{\partial^{2}}{\partial y^{2}} G_{j k}(x, t ; y, h-\zeta)\right]_{y=0} d t
\end{gathered}
$$

причем $M_{j k}(x, \zeta) \in C^{2}(0<x, \zeta<\tau), \mu_{j k}(x) \in C^{1}(0, \tau)$.

Лемма 4. Если выполняются включения

$$
\bar{\nu}_{j k}(x) \in C^{1}(0, \tau), \quad \bar{\psi}_{j k}(x) \in C[0, \tau / 2] \bigcap C^{2}(0, \tau / 2)
$$

$u \bar{\psi}_{j k}(0)=\bar{r}_{j k}(0)$, то существует единственное решение задачи Дарбу $q_{j k}^{-}(x, y) \in C\left(\bar{D}_{00}^{-}\right) \bigcap C^{2}\left(D_{00}^{-}\right)$, которое имеет вид

$$
q_{j k}^{-}(x, y)=\frac{1}{\gamma_{j k}} \int_{0}^{x+y \gamma_{j k}} \bar{\nu}_{j k}(\zeta) d \zeta+P_{j k}(x, y), \quad(x, y) \in \bar{D}_{00}^{-}
$$

əəe

$$
\begin{aligned}
P_{j k}(x, y)=-\bar{\psi}_{j k}(0)+\bar{\psi}_{j k}\left(\left(x-y \gamma_{j k}\right) / 2\right)+\bar{\psi}_{j k}\left(\left(x+y \gamma_{j k}\right) / 2\right)-B_{j k}(x, y)+ & \\
+B_{j k}\left(\left(x-y \gamma_{j k}\right) / 2\right. & \left.-\left(x-y \gamma_{j k}\right) / 2 \gamma_{j k}\right)+ \\
& +B_{j k}\left(\left(x+y \gamma_{j k}\right) / 2,-\left(x+y \gamma_{j k}\right) / 2 \gamma_{j k}\right)
\end{aligned}
$$




$$
B_{j k}(x, y)=\frac{1}{2 \gamma_{j k}} \int_{0}^{y} d t \int_{x-(y-t) \gamma_{j k}}^{x+(y-t) \gamma_{j k}} n_{j k}^{-}(\zeta, t) d \zeta .
$$

Доказательство формулы (32) следует из общего решения неоднородного уравнения (28) колебания струны

$$
\begin{aligned}
q_{j k}^{-}(x, y)=L_{j k}^{1}(x- & \left.y \gamma_{j k}\right)+L_{j k}^{2}\left(x+y \gamma_{j k}\right)+ \\
& +B_{j k}(x, y), \quad(x, y) \in \bar{D}_{00}^{-}, L_{j k}^{s}(\zeta) \in C^{2}[0, \tau] \quad(s=1,2)
\end{aligned}
$$

и краевых условий (21), (23).

Функииональное соотношение между $\bar{\omega}_{j k}(x)$ и $\bar{\nu}_{j k}(x)$, привнесенное из $D_{00}^{-}$на $y=0,0 \leqslant x \leqslant \tau$, найдем из решения (32) Дарбу, полагая в нем $y=0$ и дифференцируя соответствующее выражение:

$$
\bar{\omega}_{j k}^{\prime}(x)=\frac{1}{\gamma_{j k}} \bar{\nu}_{j k}(x)+P_{j k}^{\prime}(x, 0), \quad 0<x<\tau,
$$

где

$$
P_{j k}^{\prime}(x, 0)=\bar{\psi}_{j k}(x / 2)+\frac{1}{\gamma_{j k}} \int_{0}^{-x / 2 \gamma_{j k}} n_{j k}^{-}\left(x+t \gamma_{j k}, t\right) d t,
$$

причем

$$
P_{j k}^{\prime}(x, 0) \in C^{1}(0, \tau) .
$$

Вопрос существования решения задачи $T_{j k}(16),(19)-(21)$ в силу условий сопряжения (22)-(23) и функциональных соотношений (31), (33) сведен к разрешимости полного сингулярного интегрального уравнения нормального типа [9]

$$
\begin{gathered}
\bar{\nu}_{j k}(x)-\frac{1}{2 \tau} \int_{0}^{\tau} \bar{\nu}_{j k}(\zeta)[\operatorname{ctg}(\pi(\zeta-x) / 2 \tau)-\operatorname{ctg}(\pi(\zeta+x) / 2 \tau)] d \zeta=d_{j k}(x) \equiv \\
\equiv \gamma_{j k}\left[\mu_{j k}(x)-P_{j k}^{\prime}(x, 0)\right]-\frac{1}{\tau} \int_{0}^{\tau} \bar{\nu}_{j k}(\zeta) M_{j k}(x, \zeta) d \zeta, \quad 0<x<\tau,
\end{gathered}
$$

$d_{j k}(x) \in C^{1}(0, \tau)$, которое после преобразований и замены переменных и функций по формулам

$$
\bar{\nu}_{j k}(x)=\overline{\bar{\nu}}_{j k}(y), \quad d_{j k}(x)=\bar{d}_{j k}(y), \quad y=\cos (\pi x / \tau),
$$

примет вид

$$
\overline{\bar{\nu}}_{j k}(y)-\frac{1}{\pi} \int_{-1}^{1} \overline{\bar{\nu}}_{j k}(t) \frac{d t}{t-y}=\bar{d}_{j k}(y), \quad-1<y<1 .
$$

Индекс [9] нормального сингулярного интегрального уравнения (36) равен нулю. В силу единственности решения задачи $T_{j k}(16),(19)-(21)$ уравнение (36) однозначно обратимо в классе функций $\bar{\nu}_{j k}(y)$, удовлетворяющих условию Гельдера при $-1<y<1$, методом сингуляризации [10,11]. 
Действительно, действуя на обе части уравнения (36) оператором

$$
K v \equiv v(s)+\frac{1}{\pi} \int_{-1}^{1} v(P) \frac{d P}{P-s}
$$

используя формулу Пуанкаре--Бертрана [9] для перестановки порядка интегрирования в сингулярном повторном интеграле с ядром Коши и необходимые при этом преобразования, придем к решению уравнения (36) вида

$$
\overline{\bar{\nu}}_{j k}(y)=\frac{1}{2} \bar{d}_{j k}(y)+\frac{1}{2 \pi} \int_{-1}^{1} \bar{d}_{j k}(P) \frac{d P}{P-y}, \quad-1<y<1 .
$$

Возврат к старым переменным и функциям по формулам (35) с подстановкой правой части уравнения (34) приводит к уравнению Фредгольма [12]

$$
\bar{\nu}_{j k}(x)+\int_{0}^{\tau} \bar{\nu}_{j k}(t) W_{j k}(x, t) d t=Q_{j k}(x), \quad 0<x<\tau,
$$

где

$$
\begin{aligned}
& W_{j k}(x, t)=\frac{1}{2 \tau} M_{j k}(x, t)+ \\
& +\frac{1}{4 \tau^{2}} \int_{0}^{\tau}[\operatorname{ctg}(\pi(\zeta-x) / 2 \tau)-\operatorname{ctg}(\pi(\zeta+x) / 2 \tau)] M_{j k}(\zeta, t) d \zeta, \\
& Q_{j k}(x)=\frac{\gamma_{j k}}{2}\left[\mu_{j k}(x)-P_{j k}^{\prime}(x, 0)\right]+ \\
& +\frac{\gamma_{j k}}{4 \tau} \int_{0}^{\tau}[\operatorname{ctg}(\pi(\zeta-x) / 2 \tau)-\operatorname{ctg}(\pi(\zeta+x) / 2 \tau)]\left[\mu_{j k}(\zeta)-P_{j k}^{\prime}(\zeta, 0)\right] d \zeta
\end{aligned}
$$

причем

$$
Q_{j k}(x) \in C^{1}(0, \tau), W_{j k}(x, t) \in C^{1}(0<x, t<\tau) .
$$

Разрешимость уравнения [12] Фредгольма (37) следует из единственности решения задачи $T_{j k}$ в области $\bar{D}_{00}$.

Определив $\bar{\nu}_{j k}(x)$ из уравнения (37), найдем $\bar{\omega}_{j k}(x)$ из (31) или (33), а затем по формулам (29) и (32) получим решения $q_{j k}^{+}(x, y)$ и $q_{j k}^{-}(x, y)$ задачи Неймана-Дирихле и Дарбу соответственно в областях $D_{00}^{+}$и $D_{00}^{-}$. Таким образом, существование решения $q_{j k}(x, y)$ задачи $T_{j k}$ в области $D_{00}=$ $=D_{00}^{+} \bigcup D_{00}^{-} \bigcup I_{0}$ доказано.

Вернемся к задаче $T$ для опережающе-запаздывающего уравнения смешанного типа $(2)$ в области $D=D^{+} \bigcup D^{-} \bigcup I$. Ее решение в силу (18) и (29) имеет в области $\bar{D}^{+}=\bigcup_{j=0}^{2}\left(\bigcup_{k=0}^{1} \bar{D}_{j k}^{+}\right)$следующий вид:

$$
\begin{gathered}
\bar{U}(x, y)=\bar{U}^{+}(x, y+l h)=T\left(\begin{array}{l}
q_{00}^{+}(x, y)+(-1)^{l} q_{01}^{+}(x, y) \\
q_{10}^{+}(x, y)+(-1)^{l} q_{11}^{+}(x, y) \\
q_{20}^{+}(x, y)+(-1)^{l} q_{21}^{+}(x, y)
\end{array}\right), \\
(x, y) \in \bar{D}_{j k} \quad j=0,1,2, k, l=0,1,
\end{gathered}
$$


а в области $\bar{D}^{-}=\bigcup_{j=0}^{2} \bar{D}_{j 0}^{-}$, согласно (18) и (32), имеем

$$
\bar{U}(x, y)=\bar{U}^{-}(x, y)=T\left(\begin{array}{l}
q_{00}^{-}(x, y)+q_{01}^{-}(x, y) \\
q_{10}^{-}(x, y)+q_{11}^{-}(x, y) \\
q_{20}^{-}(x, y)+q_{21}^{-}(x, y)
\end{array}\right)
$$

Таким образом, теорема доказана.

Конкурирующие интересы. Конкурирующих интересов не имею.

Авторский вклад и ответственность. Я несу полную ответственность за предоставление окончательной версии рукописи в печать. Окончательная версия рукописи мною одобрена.

Финансирование. Исследование выполнялось без финансирования.

\section{Библиографический список}

1. Шарковский А. Н., Майстренко Ю. А., Романенко Е. Ю. Разностные уравнения и их приложения. Киев: Наук. думка, 1986. 280 с.

2. Онанов Г. Г., Скубачевский А. Л. Дифференциальные уравнения с отклоняющимися аргументами в стационарных задачах механики деформированного тела// Прикл. механика, 1979. Т. 15, № 5. С. 39-47.

3. Самарский А. А. О некоторых проблемах теории дифференциальных уравнений // Дифферени. уравн., 1980. Т. 16, № 11. С. 1925-1935.

4. Маслов В. П. Операторные методы. М.: Наука, 1973. 544 с.

5. Ганцев Ш. Х., Бахтизин Р. Н., Франц М. В., Ганцев К. Ш. Опухолевый рост и возможности математического моделирования системных процессов // Becтн. Сам. гос. техн. ун-та. Сер. Физ.-мат. науки, 2019. Т.23, №1. С. 131-151. https://doi.org/ 10.14498/vsgtu1661.

6. Беллман Р. Введение в теорию матриц. М.: Наука, 1976. 352 с.

7. Франкль Ф. И. Избранные труды по газовой динамике. М.: Наука, 1973. 712 с.

8. Зарубин А. Н. Уравнения смешанного типа с запаздывающим аргументом. Орел: ОГУ, 1997. 225 с.

9. Гахов Ф. Д. Краевые задачи. М.: Наука, 1977. 640 с.

10. Флайшер Н. М. Новый метод решения в замкнутой форме для некоторых классов сингулярных интегральных уравнений с регулярной частью// Rev. Roum. Math. Pures Appl., 1965. T. 10, № 5. C. 615-620.

11. Бабурин Ю. С. О сингуляризации сингулярных интегральных уравнений / Дифференииальные уравнения, Выпуск 10. Рязань, 1977. С. 14-24.

12. Краснов М. Л. Интегральные уравнения. М.: Наука, 1975. 303 с. 


\title{
MSC: 35M12
}

\section{Nonlocal Tricomi boundary value problem for a mixed-type differential-difference equation}

\section{(C) A. N. Zarubin}

Orel State University named after I. S. Turgenev,

95, Komsomolskaya st., Orel, 119192, Russian Federation.

\begin{abstract}
We investigate the Tricomi boundary value problem for a differentialdifference leading-lagging equation of mixed type with non-Carleman deviations in all arguments of the required function. A reduction is applied to a mixed-type equation without deviations. Symmetric pairwise commutative matrices of the coefficients of the equation are used. The theorems of uniqueness and existence are proved. The problem is unambiguously solvable.
\end{abstract}

Keywords: mixed-type equation, differential-difference equation, integral equation, singular integral equation, concentrated lag and lead.

Received: $5^{\text {th }}$ November, $2020 /$ Revised: $13^{\text {th }}$ February, $2021 /$ Accepted: 22 ${ }^{\text {nd }}$ February, 2021 / First online: 10 ${ }^{\text {th }}$ March, 2021

Competing interests. I have no competing interests.

Authors' contributions and responsibilities. I take full responsibility for submitting the final manuscript in print. I approved the final version of the manuscript.

Funding. This research received no specific grant from any funding agency in the public, commercial, or not-for-profit sectors.

\section{References}

1. Sharkovsky A. N., Maistrenko Yu. L., Romanenko E. Yu Difference Equations and Their Applications, Mathematics and Its Applications, vol. 250. Dordrecht, Kluwer Academic Publ., 1993, xii+358 pp. https://doi.org/10.1007/978-94-011-1763-0.

2. Onanov G. G., Skubachevskii A. L. Differential equations with displaced arguments in stationary problems in the mechanics of a deformable body, Sov. Appl. Mech., 1979, vol. 15, no. 5, pp. 391-397. https://doi.org/10.1007/BF01074069.

3. Samarskii A. A. Some problems of the theory of differential equations, Differ. Uravn., 1980, vol. 16, no. 11, pp. 1925-1935 (In Russian).

\section{Research Article}

(ㅇ)(i) The content is published under the terms of the Creative Commons Attribution 4.0 International License (http://creativecommons.org/licenses/by/4.0/)

Please cite this article in press as:

Zarubin A. N. Nonlocal Tricomi boundary value problem for a mixed-type differential-difference equation, Vestn. Samar. Gos. Tekhn. Univ., Ser. Fiz.-Mat. Nauki [J. Samara State Tech. Univ., Ser. Phys. Math. Sci.], 2021, vol. 25, no. 1, pp. 35-50. https://doi.org/10.14498/vsgtu1835 (In Russian).

\section{Author's Details:}

Aleksandr N. Zarubin (10) https://orcid.org/0000-0002-0611-5752

Dr. Phys. \& Math. Sci., Professor; Head of Dept.; Dept. of Mathematical Analysis and Differential Equations; e-mail: aleks_zarubin@mail.ru; matdiff@yandex.ru 
4. Maslov V. P. Operatornye metody [Operational Methods]. Moscow, Nauka, 1973, 544 pp. (In Russian)

5. Gantsev Sh., Bakhtizin R. N., Frants M. V., Gantsev K. Sh. Tumor growth and mathematical modeling of system processes, Vestn. Samar. Gos. Tekhn. Univ., Ser. Fiz.-Mat. Nauki [J. Samara State Tech. Univ., Ser. Phys. Math. Sci.], 2019, vol. 23, no.1, pp. 131-151 (In Russian). https://doi.org/10.14498/vsgtu1661.

6. Bellman R. Introduction to matrix analysis, Society for Industrial and Applied Mathematics, vol. 19. Philadelphia, PA, 1997, xxviii +403 pp.

7. Frankl F. I. Izbrannye trudy po gazovoi dinamike [Selected Works on Gas Dynamics]. Moscow, Nauka, 1973, 712 pp. (In Russian)

8. Zarubin A. N. Uravneniia smeshannogo tipa s zapazdyvaiushchim argumentom [Mixed-Type Equations with Retarded Argument]. Orel, Orel State Univ., 1997, 225 pp. (In Russian)

9. Gakhov F. D. Boundary Value Problems, International Series of Monographs in Pure and Applied Mathematics, vol.85. Oxford, Pergamon Press, 1966, xix+564 pp. https://doi. org/10.1016/C2013-0-01739-2.

10. Flaysher N. M. A new closed-form solution method for some classes of singular integral equations with a regular part, Rev. Roum. Math. Pures Appl., 1965, vol.10, no. 5, pp. 615620 (In Russian).

11. Baburin Yu. S. On the singularization of singular integral equations, In: Differentsial'nye uravneniia [Differential Equations], Issue 10. Ryazan, 1977, pp. 14-24 (In Russian).

12. Krasnov M. L. Integral'nye uravneniia [Integral Equations]. Moscow, Nauka, 1975, 303 pp. (In Russian) 